\title{
Avaliação do desenvolvimento do setor agropecuário no Brasil: período de 1970 a $2000^{1}$
}

\author{
Clailton Ataídes de Freitas ${ }^{2}$ \\ Carlos José Caetano Bacha ${ }^{3}$ \\ Daniele Maria Fossatti ${ }^{4}$
}

\section{Resumo}

Este artigo analisa o processo de desenvolvimento econômico do setor agropecuário no Brasil no período de 1970 a 2000, destacando a sua desigualdade entre os estados da federação. Procura-se ressaltar a influência do estoque de capital físico (medido pela potência dos tratores c.v.) e do capital humano sobre o desenvolvimento agropecuário dos estados brasileiros. Com relação ao capital humano verifica-se que, de um modo geral, o nível de qualificação dos agricultores brasileiros é muito baixo, mesmo nos estados brasileiros mais desenvolvidos como São Paulo e Rio Grande do Sul, por exemplo. O menor nível de educação está nos estados nordestinos. Isso explica, em parte, o relativo atraso da agropecuária dessa região em relação à agropecuária dos demais estados. Constatase, também, que a intensificação do uso de capital na agropecuária no Brasil vem crescendo em todos os estados a taxas superiores a $5 \%$ a.a. No entanto, pode-se verificar que foram justamente os estados nordestinos os que apresentaram, em termos relativos, as menores taxas de crescimento no uso de capital. Há, portanto, desequilíbrios regionais na agropecuária que não estão reduzindo por si próprios. No final, o artigo sugere medidas para diminuir as diferenças supracitadas.

Palavras-chave: Agropecuária - Brasil; Desenvolvimento econômico; Disparidades regionais; Capital (Economia); Capital humano.

\section{Abstract}

The evaluation of agriculture growth in Brazil: time period from 1970 to 2003

This paper analyzes Brazilian agricultural development from 1970 to 2000, highlighting how this process took place differently among Brazil's states. Special attention is paid to the role of physical and human capital over Brazilian states' agricultural development process. The main findings are: first, all Brazilian states show low levels of human capital at their agricultural sector; especially the Northeastern states what have the nationwide lowest level of formal education among their workers. The latter partially explains the nationwide lowest rank of Northeastern states' agricultural sector. Second, capital use intensification has risen by $5 \%$ per year, in average. However, the Northeastern states show lower increase for this parameter. Regional differences into the agricultural sector are evident and they are not disappearing by themselves. At the end, the paper suggests some policies that can reduce these regional differences.

Key words: Agricultural development; Regional imbalances; Capital use intensification; Human capital.

JEL O13, Q10, R12.

(1) Trabalho recebido em novembro de 2005 e aprovado em fevereiro de 2007.

(2) Professor Adjunto do Departamento de Ciências Econômicas e do Programa de Pós-Graduação do Mestrado em Integração Latino-Americana (MILA) da Universidade de Santa Maria (UFSM), RS. E-mail: $<$ caf@ccsh.ufsm.br>.

(3) Professor titular da Escola Superior de Agricultura Luiz de Queiroz (ESALQ) da Universidade de São Paulo (USP). E-mail: <cjcbacha@esalq.usp.br>.

(4) Mestranda do MILA/UFSM. E-mail: <danimaria.fossatti@bol.com.br>. 


\section{Introdução}

Inicialmente, é necessário distinguir crescimento econômico e desenvolvimento econômico. Entende-se como crescimento econômico o processo de aumento do produto (interno ou nacional) de um país ou setor. Normalmente, crescimento econômico é medido pela evolução do produto total ou do produto per capita de um país ou setor.

Existem várias definições de desenvolvimento econômico, das quais duas merecem destaque. Segundo Araújo E Schuh (1995, p. 10-11), “... para a Comissão Econômica para a América Latina (Cepal), desenvolvimento econômico pode ser conceituado como um processo dinâmico de mudança estrutural da economia, objetivando proporcionar à maioria da população: (a) maiores níveis de consumo; (b) possibilidade de chegar a idades mais avançadas; (c) maiores recursos para educação; e (d) redução da jornada de trabalho". Para Celso Furtado (1961), citado por Araújo e Schuh (1995, p. 10), "desenvolvimento econômico consiste na introdução de novas combinações de fatores de produção visando aumentar a produtividade do trabalho. Quando cresce a produtividade do trabalho aumenta o produto social, isto é, a quantidade de bens e serviços à disposição da sociedade. Por outro lado, o crescimento da renda provoca nos consumidores reações que aumentam a procura e modificam sua estrutura. $\mathrm{O}$ aumento e a diversificação da procura fazem com que também se modifique a estrutura da produção".

A partir das definições da Cepal e de Celso Furtado, pode-se conceber desenvolvimento econômico como um processo de mudança estrutural da economia (na qual se destacam novas combinações de uso de fatores) que implicam melhoria do bem-estar da população, a qual se manifesta através de maior nível de renda per capita, melhor nível de educação e melhor nível de saúde.

$\mathrm{O}$ crescimento econômico é condição necessária e não suficiente para haver o desenvolvimento econômico. $\mathrm{Na}$ análise da evolução da agropecuária brasileira tem sido dada muita atenção ao seu crescimento econômico, avaliado pelo crescimento do PIB ou da produção, mas não ao seu desenvolvimento econômico, o qual pode apresentar diferenças entre regiões e, assim, implicar um repensar da política econômica.

É baseado no acima exposto que o objetivo deste trabalho é analisar o desenvolvimento econômico, em nível de estado da federação, do setor agropecuário brasileiro no período de 1970 a 2000. Especificamente, procura-se avaliar a diferença de produto per capita e por trabalhador na agropecuária entre os estados brasileiros e associar essa diferença com o processo desigual de tecnificação da agropecuária brasileira e de evolução do estoque de capital humano na agropecuária dos diversos estados brasileiros. 
O período abordado por este estudo é caracterizado pela intensificação do progresso tecnológico na agropecuária brasileira. O salto tecnológico neste setor provocou profundas transformações na maneira de produzir do agricultor em todas as regiões brasileiras. Esta inovação foi levada a cabo através do uso de insumos e equipamentos modernos. A maneira tradicional de produzir do agricultor brasileiro foi, também, se alterando, de modo a atender às exigências do mercado com relação a produtos com maior qualidade e com custos menores. No entanto, tal inovação se fez de maneira diferenciada em termos de produtos e estados do Brasil.

Diversos trabalhos já analisaram, para parte do período aqui considerado, o crescimento da agropecuária brasileira, mas não o seu desenvolvimento. Podem ser citados os trabalhos de Barros e Graham (1978), Rezende (1989), Mello (1990), Gasques e Villa Verde (1990), Silva (1991), Ferreira Filho (1994), Bacha e Rocha (1998), Staduto e Freitas (2001), Freitas e Bacha (2002), entre outros. Esses trabalhos dão maior atenção à evolução diferenciada da agropecuária em termos de produtos, e não entre estados e dedicando-se pouco à questão da tecnificação e do capital humano. Há trabalhos que analisaram a evolução da produtividade da agropecuária brasileira, procurando quantificá-la, mas não avaliando como sua tecnificação foi diferenciada entre estados, caso de Arnade (1992), Ávila et al. (1995) e Gasques e Conceição (1997; 2001).

A principal contribuição do presente trabalho é apresentar os dados que evidenciam o desenvolvimento desigual da agropecuária entre os estados brasileiros e demonstrar como o estoque de capital humano explica essa diferenciação ao longo do período analisado.

A metodologia utilizada no trabalho é a análise tabular de dados secundários coletados em diferentes publicações do IBGE, bem como da literatura disponível. A partir desses dados, alguns indicadores de crescimento e desenvolvimento econômico, ainda não disponíveis na literatura, são calculados. $\mathrm{O}$ presente trabalho está composto de três itens, incluindo esta introdução. O item 1 analisa a diferença de produto agropecuário per capita e por trabalhador entre os estados brasileiros e sua evolução; também se avaliam a tecnificação da agropecuária, o capital humano e a distribuição regional da produção agrícola per capita (que são aqui considerados como indicadores de desenvolvimento econômico). Procura-se ressaltar as diferenças desses indicadores entre os estados brasileiros. O último item traz as conclusões do trabalho.

\section{Análise da diferença estadual de desenvolvimento do setor agropecuário brasileiro}

Uma radiografia estanque do mundo mostraria que, em termos comparativos, existem em um extremo os países ricos, com as suas economias 
bem estruturadas, e no outro extremo os países pobres com dificuldades de manter taxas de crescimento econômico acima do crescimento demográfico. Entre os dois extremos está a maioria dos países com suas economias em transição. São os chamados países em desenvolvimento, que se caracterizaram por apresentar elevadas taxas de crescimento econômico, acima de 5\% ao ano nas últimas décadas (ver, por exemplo, Jones, 1999, p. 4). No entanto, esses países ainda convivem com problemas relacionados ao déficit público, endividamento externo e interno, inflação, concentração de renda, entre outros. Essas variáveis em conjunto contribuem para determinar o nível de vulnerabilidade das economias desses países.

Os desequilíbrios regionais, que caracterizam o processo de acumulação no sistema capitalista, resultam em parte do movimento do capital. Este se aloca em algumas regiões, tentando aproveitar-se de algumas variáveis que são fundamentais para a geração de economias de escala, tais como: disponibilidade de mão-de-obra qualificada e no volume requisitado, proximidade dos centros consumidores, facilidade de transporte das matérias-primas e dos produtos finais e infra-estrutura eficiente e adequada às exigências dos investimentos.

Trazendo a discussão da desigualdade econômica para o Brasil, percebe-se que, de um lado, estão alguns estados que poderão ser considerados ricos, levando em conta a realidade do país, e no outro extremo estão os estados pobres. Na zona intermediária se encontram os estados com economias em transição. Vários trabalhos já analisaram essa desigualdade (ver, por exemplo, Lavinas et al., 1993, e Barros et al., 1997). Por essa razão, o presente estudo procurou apresentar alguns indicadores econômicos, já consagrados na literatura de desenvolvimento econômico, com a finalidade de utilizá-los como referência para estudar as diferenças no comportamento econômico recente da agropecuária nos estados brasileiros.

No presente trabalho, optou-se pela utilização do valor da produção agropecuária per capita e por equivalente-homem ${ }^{5}$ (força de trabalho) como indicador de desenvolvimento, em vez de se usar, como é comum nas análises econômicas, o PIB per capita, ou outros indicadores sociais, como mortalidade infantil, expectativa de vida e analfabetismo, por exemplo.

A Tabela 1 apresenta os dados absolutos das três variáveis que são úteis para comparar o crescimento e o desenvolvimento econômicos entre os estados brasileiros. Por exemplo, as colunas de 2 a 4 mostram a relação entre o valor da produção e o equivalente-homem. Trata-se de uma medida agregada de produtividade do trabalho. Ao calcular a taxa de crescimento das variáveis apresentadas na Tabela 1, produziu-se a Tabela 2.

(5) A fórmula de cálculo do número de equivalentes-homem está em Silva e Kageyama (1983). 
Tabela 1

Valor da produção (VP), em reais de março de 2004, por equivalente-homem $(\mathrm{EH})^{(1)}$ e per capita; e taxa de participação da população rural

\begin{tabular}{|c|c|c|c|c|c|c|c|c|c|}
\hline \multirow{2}{*}{ UF } & \multicolumn{3}{|c|}{ VP por EH } & \multicolumn{3}{|c|}{ VP per capita } & \multicolumn{3}{|c|}{ Eh/pop. rural } \\
\hline & 1970 & 1996 & 2000 & 1970 & 1996 & 2000 & 1970 & 1996 & 2000 \\
\hline RO & 2418,058 & 5198,714 & 3527,36 & 741,33 & 2618,74 & 1453,78 & 0,31 & 0,50 & 0,41 \\
\hline $\mathrm{AC}$ & 1960,872 & 3935,208 & 3034,317 & 610,84 & 1627,42 & 1038,11 & 0,31 & 0,41 & 0,34 \\
\hline $\mathrm{AM}$ & 1886,798 & 942,2272 & 1730,699 & 729,69 & 392,38 & 611,60 & 0,39 & 0,42 & 0,35 \\
\hline $\mathrm{RR}$ & 5258,193 & 5884,488 & 4668,626 & 1425,31 & 2124,20 & 1214,03 & 0,27 & 0,36 & 0,26 \\
\hline PA & 1387,623 & 3412,931 & 3810,667 & 502,15 & 897,53 & 855,23 & 0,36 & 0,26 & 0,22 \\
\hline $\mathrm{AP}$ & 3178,176 & 2817,242 & 3219,742 & 485,51 & 742,40 & 710,81 & 0,15 & 0,26 & 0,22 \\
\hline MA & 216,121 & 1075,463 & 915,2592 & 86,94 & 460,49 & 355,46 & 0,40 & 0,43 & 0,39 \\
\hline PI & 532,3582 & 1427,079 & 892,696 & 193,15 & 670,73 & 380,75 & 0,36 & 0,47 & 0,43 \\
\hline $\mathrm{CE}$ & 541,234 & 1262,041 & 1156,16 & 176,49 & 576,10 & 481,65 & 0,33 & 0,46 & 0,42 \\
\hline $\mathrm{RN}$ & 816,6941 & 2096,782 & 1238,199 & 261,95 & 825,42 & 437,53 & 0,32 & 0,39 & 0,35 \\
\hline PB & 917,9209 & 3296,595 & 1382,908 & 315,29 & 1246,16 & 463,28 & 0,34 & 0,38 & 0,34 \\
\hline $\mathrm{PE}$ & 1211,139 & 2432,932 & 1386,046 & 464,82 & 1016,81 & 535,37 & 0,38 & 0,42 & 0,39 \\
\hline $\mathrm{AL}$ & 1617,47 & 3242,512 & 3666,798 & 573,75 & 1203,28 & 1260,19 & 0,35 & 0,37 & 0,34 \\
\hline SE & 1174,643 & 2120,203 & 1717,871 & 517,96 & 1134,19 & 855,98 & 0,44 & 0,53 & 0,50 \\
\hline $\mathrm{BA}$ & 1563,737 & 2348,782 & 2644,484 & 571,93 & 997,36 & 1008,80 & 0,37 & 0,42 & 0,38 \\
\hline MG & 3594,593 & 4474,465 & 6085,44 & 1105,43 & 2153,25 & 2566,90 & 0,31 & 0,48 & 0,42 \\
\hline ES & 5413,71 & 16777,64 & 7867,617 & 1525,97 & 8000,67 & 2636,16 & 0,28 & 0,48 & 0,34 \\
\hline RJ & 3678,036 & 5921,309 & 5380,074 & 778,90 & 1547,80 & 1229,80 & 0,21 & 0,26 & 0,23 \\
\hline SP & 5278,908 & 14619,08 & 14115,55 & 1870,85 & 5243,92 & 4479,51 & 0,35 & 0,36 & 0,32 \\
\hline PR & 4306,575 & 6801,036 & 11161,82 & 1524,57 & 3673,27 & 5437,59 & 0,35 & 0,54 & 0,49 \\
\hline $\mathrm{SC}$ & 2655,202 & 5268,718 & 6717,039 & 926,97 & 2291,35 & 2659,22 & 0,35 & 0,43 & 0,40 \\
\hline RS & 4297,096 & 9088,23 & 9596,979 & 1634,08 & 4946,04 & 4826,86 & 0,38 & 0,54 & 0,50 \\
\hline MT & 2978,124 & 6639,655 & 11695,58 & 966,52 & 5609,46 & 8651,82 & 0,32 & 0,84 & 0,74 \\
\hline GO & 2261,772 & 4599,267 & 7249,122 & 614,58 & 4056,75 & 5480,39 & 0,27 & 0,88 & 0,76 \\
\hline $\mathrm{DF}$ & 2797,207 & 10914,51 & 18675,64 & 769,53 & 1109,98 & 1730,80 & 0,28 & 0,10 & 0,09 \\
\hline
\end{tabular}

(1) A força de trabalho empregada na agricultura foi convertida em equivalente-homem/ano, que diz respeito a um homem adulto trabalhando durante 300 dias por ano. Se o leitor desejar obter mais detalhes, ver Freitas (2001). Para o ano de 2000, não há dados disponíveis para calcular o total de equivalentes-homem. Dessa forma, tomou-se a taxa de crescimento da população no meio rural, dividida por sexo, entre os anos 1996 e 2000, e multiplicou-se pelo quantum da população economicamente ativa em 1996. Esse novo dado tornou-se a proxy do total de equivalentes-homem para 2000 , feitos seus devidos cálculos.

Fonte: Cálculos dos autores com base em dados do Anuário Estatístico do Brasil (vários anos).

Na Tabela 2, pode-se constatar que a produtividade do trabalho na agropecuária em alguns estados vem crescendo a taxas elevadas. Por exemplo, no Distrito Federal, Mato Grosso, Goiás e Maranhão, o crescimento da produtividade do trabalho, entre 1970 e 2000, foi acima de 3,95\% ao ano. Se a análise voltar-se apenas para os quatro últimos anos da pesquisa (1996 a 2000), observa-se crescimento mais acentuado da produtividade do trabalho nos estados do Distrito Federal (14,37\% a.a.), Goiás (12,05\% a.a.), Mato Grosso (15,20\% a.a.), Paraná (13,19\% a.a.) e Amazonas (16,42\% a.a.). Apenas para Amazonas e Roraima observam-se valores negativos para a taxa de crescimento do valor da produção 
por equivalente-homem (VP por EH) no período de 1970 a 2000. Entretanto, para o período de 1996 a 2000, observam-se taxas negativas para a maioria dos estados do Nordeste e para alguns estados da Região Sudeste, como descrito na Tabela 2.

Tabela 2

Taxa de crescimento anual, em \% do valor da produção por equivalente-homem e per capita, e da taxa de participação da população rural

\begin{tabular}{|c|c|c|c|c|c|c|c|c|c|}
\hline \multirow{2}{*}{$\mathrm{UF}$} & \multicolumn{3}{|c|}{ VP por EH } & \multicolumn{3}{|c|}{ VP per capita } & \multicolumn{3}{|c|}{ EH/População rural } \\
\hline & $70-96$ & $96-00$ & $70-00$ & $70-96$ & $96-00$ & $70-00$ & $70-96$ & $96-00$ & $70-00$ \\
\hline RO & 2,99 & $-9,24$ & 1,27 & 4,97 & $-13,68$ & 2,27 & 1,93 & $-4,89$ & 0,99 \\
\hline $\mathrm{AC}$ & 2,72 & $-6,29$ & 1,47 & 3,84 & $-10,63$ & 1,78 & 1,10 & $-4,63$ & 0,31 \\
\hline $\mathrm{AM}$ & $-2,64$ & 16,42 & $-0,29$ & $-2,36$ & 11,74 & $-0,59$ & 0,29 & $-4,02$ & $-0,30$ \\
\hline $\mathrm{RR}$ & 0,43 & $-5,62$ & $-0,40$ & 1,55 & $-13,05$ & $-0,53$ & 1,11 & $-7,87$ & $-0,14$ \\
\hline $\mathrm{PA}$ & 3,52 & 2,79 & 3,42 & 2,26 & $-1,20$ & 1,79 & $-1,22$ & $-3,89$ & $-1,58$ \\
\hline $\mathrm{AP}$ & $-0,46$ & 3,39 & 0,04 & 1,65 & $-1,08$ & 1,28 & 2,12 & $-4,33$ & 1,23 \\
\hline TO & & & & & 8,04 & & & & \\
\hline MA & 6,37 & $-3,95$ & 4,93 & 6,62 & $-6,27$ & 4,81 & 0,24 & $-2,41$ & $-0,12$ \\
\hline PI & 3,87 & $-11,07$ & 1,74 & 4,90 & $-13,20$ & 2,29 & 1,00 & $-2,40$ & 0,54 \\
\hline $\mathrm{CE}$ & 3,31 & $-2,17$ & 2,56 & 4,66 & $-4,38$ & 3,40 & 1,30 & $-2,26$ & 0,82 \\
\hline $\mathrm{RN}$ & 3,69 & $-12,34$ & 1,40 & 4,51 & $-14,67$ & 1,72 & 0,79 & $-2,66$ & 0,32 \\
\hline $\mathrm{PB}$ & 5,04 & $-19,52$ & 1,38 & 5,43 & $-21,91$ & 1,29 & 0,37 & $-2,97$ & $-0,08$ \\
\hline $\mathrm{PE}$ & 2,72 & $-13,12$ & 0,45 & 3,06 & $-14,82$ & 0,47 & 0,33 & $-1,95$ & 0,02 \\
\hline $\mathrm{AL}$ & 2,71 & 3,12 & 2,77 & 2,89 & 1,16 & 2,66 & 0,17 & $-1,90$ & $-0,11$ \\
\hline SE & 2,30 & $-5,12$ & 1,28 & 3,06 & $-6,79$ & 1,69 & 0,75 & $-1,76$ & 0,41 \\
\hline $\mathrm{BA}$ & 1,58 & 3,01 & 1,77 & 2,16 & 0,29 & 1,91 & 0,58 & $-2,64$ & 0,14 \\
\hline MG & 0,85 & 7,99 & 1,77 & 2,60 & 4,49 & 2,85 & 1,74 & $-3,24$ & 1,06 \\
\hline ES & 4,45 & $-17,25$ & 1,25 & 6,58 & $-24,24$ & 1,84 & 2,04 & $-8,44$ & 0,58 \\
\hline $\mathrm{RJ}$ & 1,85 & $-2,37$ & 1,28 & 2,68 & $-5,59$ & 1,53 & 0,81 & $-3,30$ & 0,26 \\
\hline SP & 4,00 & $-0,87$ & 3,33 & 4,04 & $-3,86$ & 2,95 & 0,05 & $-3,02$ & $-0,37$ \\
\hline PR & 1,77 & 13,19 & 3,23 & 3,44 & 10,30 & 4,33 & 1,64 & $-2,55$ & 1,07 \\
\hline $\mathrm{SC}$ & 2,67 & 6,26 & 3,14 & 3,54 & 3,79 & 3,58 & 0,85 & $-2,32$ & 0,42 \\
\hline RS & 2,92 & 1,37 & 2,71 & 4,35 & $-0,61$ & 3,68 & 1,39 & $-1,95$ & 0,94 \\
\hline MS & & & & & 12,47 & & & & \\
\hline MT & 3,13 & 15,20 & 4,67 & 7,00 & 11,44 & 7,58 & 3,75 & $-3,27$ & 2,78 \\
\hline GO & 2,77 & 12,05 & 3,96 & 7,53 & 7,81 & 7,57 & 4,63 & $-3,78$ & 3,47 \\
\hline $\mathrm{DF}$ & 5,38 & 14,37 & 6,53 & 1,42 & 11,75 & 2,74 & $-3,76$ & $-2,30$ & $-3,56$ \\
\hline
\end{tabular}

Fonte: Percentagens calculadas com base nos dados da Tabela 1. A taxa de crescimento é o valor de i, em percentagem, dado pela fórmula $V a=V o(1+i)^{n}$.

A quinta, a sexta e a sétima colunas da Tabela 1 evidenciam o valor da produção agropecuária per capita em 1970, 1996 e 2000, respectivamente. Tratase de outro indicador de desenvolvimento econômico, o qual permite avaliar a capacidade de consumo potencial da população rural. Tal como sugerido pelo conceito de Celso Furtado (apresentado na introdução deste artigo), supõe-se que o bem-estar da população é maior quanto maior é sua capacidade de consumo.

A maioria dos estados apresentou taxa de crescimento para o valor da produção per capita acima de 3\% ao ano, no período de 1970 a 1996, com 
destaque para Goiás, Mato Grosso e Maranhão, cujas taxas de crescimento superaram 6,5\% ao ano. Contudo, o valor da produção per capita no Maranhão era 11,4 vezes menor do que o verificado em São Paulo em 1996. Em média, os valores da produção per capita dos estados das Regiões Norte e Nordeste foram, aproximadamente, seis vezes menores do que o obtido por São Paulo. Entretanto, no período de 1996 a 2000 há a reversão na tendência de crescimento do valor da produção per capita. De acordo com os dados do Anuário Estatístico do Brasil, nesse período todos os estados da Região Norte (exceto Amazonas) e a maioria dos estados do Nordeste brasileiro apresentaram crescimento negativo, com altas taxas em valores absolutos, enquanto estados do Centro-Oeste apresentam taxas positivas e elevadas de crescimento do VP per capita.

De acordo com os valores da produção per capita (Tabela 1), verifica-se que o maior bem-estar da população no meio rural encontra-se nas Regiões Sudeste (com exceção do Rio de Janeiro), Sul e Centro-Oeste (excluindo o Distrito Federal). Nessas regiões, o valor da produção per capita superou os R $\$ 1.600,00$, em 1996, e em 2000 alcançou valores acima de $\mathrm{R} \$ 2.600,00$ (a preços de março de 2004). A Região Norte apresenta certa disparidade: os Estados de Roraima, Acre e Rondônia apresentam VP per capita acima de R\$1.000,00, ao passo que Amazonas, Pará e Amapá possuem valor da produção per capita inferior a R\$ 900,00, tanto para o ano de 1996 quanto para 2000. Para os estados do Nordeste, apenas a Bahia, Sergipe e Alagoas obtiveram valores de produção per capita acima de $\mathrm{R} \$ 800,00$ para o ano de 2000, enquanto os outros estados obtiveram valores inferiores a $\mathrm{R} \$ 600,00$.

A oitava, a nona e a décima colunas da Tabela 1 apresentam as proporções das pessoas que trabalham sobre as que vivem no meio rural (EH/população rural). Quanto mais próximo de um for esse quociente, menor é a proporção de crianças e idosos sobre a quantidade total de equivalentes-homem e também menor é o desemprego da mão-de-obra no campo. Somente em seis estados a proporção de EH sobre a população rural superou os $49 \%$ em 1996. Nos outros estados, à exceção do Distrito Federal, a taxa de ocupação da mão-de-obra ficou entre 25 e $50 \%$. Para o ano de 2000, o número de estados com taxa de ocupação superior a 49\% caiu para quatro (Sergipe, Rio Grande do Sul, Mato Grosso e Goiás).

Com exceção ao Estado do Pará e do Distrito Federal, houve aumento da taxa de ocupação da mão-de-obra nos estados entre 1970 e 1996. Entretanto, fenômeno inverso ocorreu para o período de 1996 a 2000, no qual todos os estados obtiveram taxas negativas de crescimento da variável EH/população rural, como mostra a Tabela 2. Há, portanto, aumento do desemprego e subocupação da mãode-obra no campo a partir da $2^{2}$ metade da década de 1990. Isso está associado ao aumento da mecanização e racionalização da produção que tem ocorrido. 
A Tabela 3 apresenta a relação capital/trabalho na agropecuária brasileira ${ }^{6}$. Em uma típica função de produção (excluindo o estágio III), quanto maior for a quantidade de capital por trabalhador a ser utilizada, maior será o produto por trabalhador a ser obtido. Observa-se na quarta coluna da Tabela 3 que, em 1995/96, os estados do Sudeste, Sul e Centro-Oeste eram os que tinham maior relação $K / L$. Isso explica o fato de eles também serem os que tinham maior valor da produção por equivalente-homem no mesmo ano (como elucidado na Tabela 1). O coeficiente de correlação entre a variável K/L e o VP por equivalente-homem para o Brasil como um todo em 1996 foi de 0,97 para a Região Sul, 0,96 para o Centro-Oeste, 0,71 para a Região Norte e 0,76 para o Nordeste. Mas foi pouco expressivo para a Região Sudeste $(0,44)$, no qual há grande diferença tecnológica de São Paulo em relação aos três outros estados da região.

Tabela 3

Evolução da relação entre capital e trabalho na agropecuária brasileira

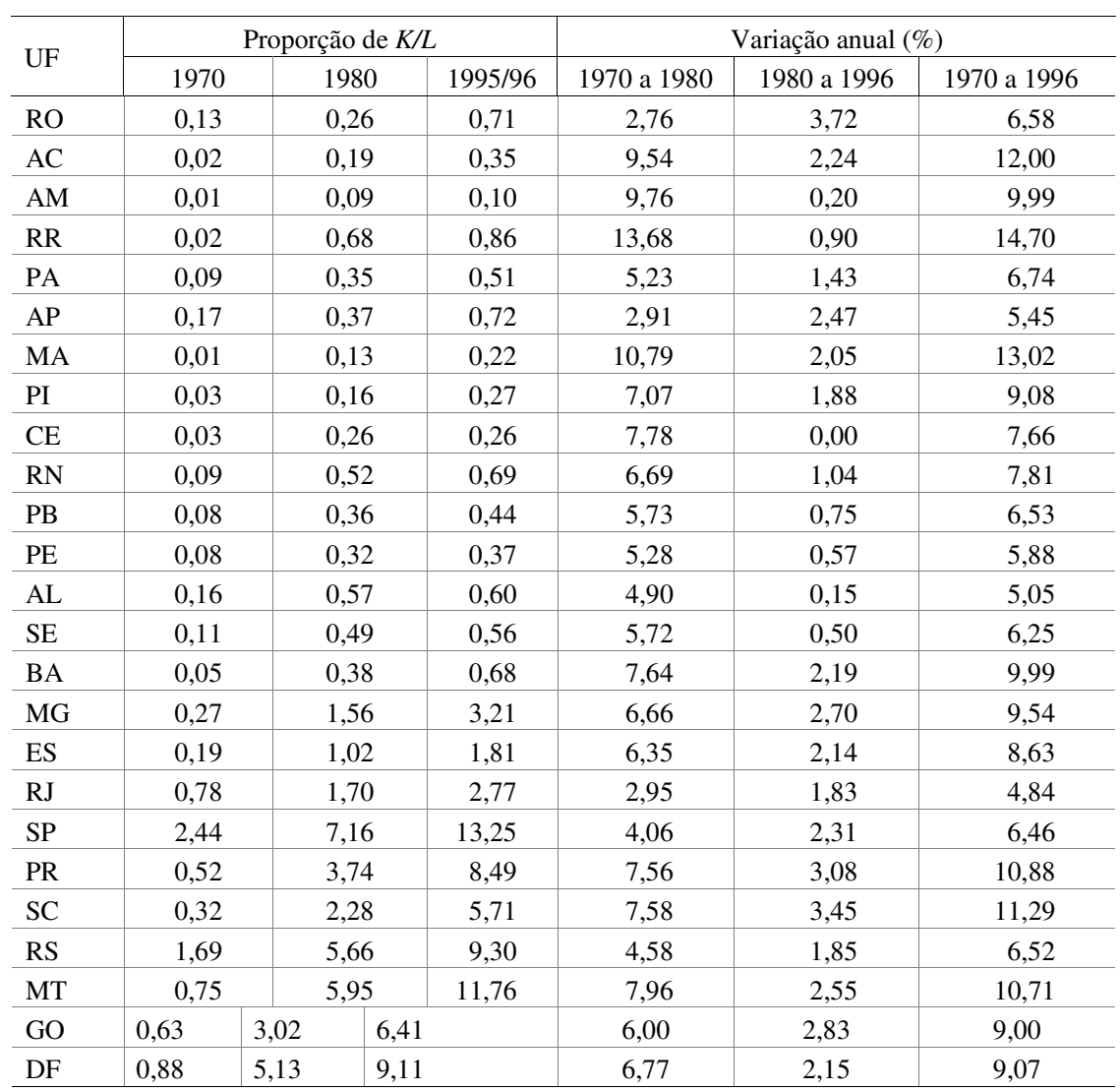

Fonte: Censo Agropecuário do Brasil (Vários anos).

(6) A proxy utilizada para o capital foi a potência dos tratores, medida em número de cavalos (c.v.) em cada estado. $\mathrm{O} L$ refere-se ao número de equivalentes-homem (EH). 
Examinando a evolução da relação capital/trabalho $(K / L)$, fica patente o processo de intensificação do uso de capital na agropecuária brasileira. Em 1970, somente São Paulo e Rio Grande do Sul possuíam relação $K / L$ maior do que 1. Observa-se que a relação $K / L$ era em média, em 1970, muito baixa para os estados da Região Norte e Nordeste. Nos anos de 1980 e 1995/96, todos os estados das Regiões Sudeste, Sul e Centro-Oeste já apresentavam a proporção $K / L$ superior a 1. Evidenciando, assim, que o processo de tecnificação, medido pela mecanização do setor agropecuário, tendeu a se concentrar nessas regiões em detrimento das demais.

Entre 1970 e 1980, a expansão da tratorização na agropecuária brasileira foi muita elevada. Na grande maioria dos estados do Brasil verificou-se que a taxa geométrica de crescimento da relação $K / L$ foi superior a $5 \%$ ao ano. No período de 1980 a 1996, verificou-se que o ritmo de mecanização do setor agropecuário diminuiu. As taxas de variação percentuais da proporção $K / L$ diminuíram bastante, conforme se pode perceber através da sexta coluna da Tabela 3, e comparando com as taxas da quinta coluna. Resultado semelhante foi obtido por Barros (1999).

Considerando todo o período de 1970 a 1996, é possível constatar que a relação $K / L$ vem crescendo em todos os estados brasileiros a taxa superior a $5 \%$ ao ano, exceto no Rio de Janeiro (ver a última coluna da Tabela 3). Em vários outros estados, essa taxa foi superior a $10 \%$ ao ano, como nos casos do Paraná, Santa Catarina e Maranhão, por exemplo. No entanto, pode-se facilmente perceber que os estados da Região Nordeste, de modo geral, foram os que apresentaram as menores taxas de crescimento da tecnificação. Isso é o resultado material da discriminação da região em termos da distribuição do crédito rural e do baixo nível de qualificação dos agricultores. Este último aspecto é estudado a seguir.

Considerando a totalidade dos setores da economia brasileira, pode-se perceber (ver Tabela 4) que a média dos anos de estudo do trabalhador brasileiro era de aproximadamente três anos em 1970 e 6,81 anos em 2000. Esse nível de educação média apresentou crescimento da ordem de $125 \%$ entre esses dois anos.

Ainda analisando os dados da Tabela 4, verifica-se que o setor agropecuário brasileiro apresentou, em 1970, em torno de um ano de educação média de seus trabalhadores. Esse número representa apenas $33 \%$ do verificado no Brasil, para o mesmo ano. Em 1980 com o setor agropecuário apresentando uma média de 1,51 ano de estudo, contra 4,37 anos de estudo para o conjunto de trabalhadores do Brasil, constata-se que, apesar dos avanços nos níveis de educação no meio rural, o número médio de anos de educação formal no setor agropecuário continuou representando em torno de $35 \%$ da média brasileira. Essa diferença se reduz bastante em 1991, quando a educação média do trabalhador no setor agropecuário atinge $45 \%$ do valor da educação média do conjunto de trabalhadores do Brasil. Em 2000, a educação média do trabalhador no setor agropecuário atinge $51 \%$ da educação média do conjunto de trabalhadores do Brasil. 
Tabela 4

Anos médios de estudo dos trabalhadores com 10 anos ou mais de idade

\begin{tabular}{|c|c|c|c|c|c|c|c|c|}
\hline \multirow{2}{*}{ UF } & \multicolumn{4}{|c|}{ Todos os setores } & \multicolumn{4}{|c|}{ Setor agropecuário } \\
\hline & $1970^{\text {(a) }}$ & $1980^{\text {(a) }}$ & $1991^{\text {(a) }}$ & $2000^{(b)}$ & $1970^{\text {(a) }}$ & $1980^{\text {(a) }}$ & $1991^{\text {(a) }}$ & $2000^{(\mathrm{b})}$ \\
\hline RO & 2,32 & 3,06 & 5,10 & 6,26 & 0,77 & 1,64 & 2,86 & 3,88 \\
\hline $\mathrm{AC}$ & 1,35 & 2,18 & 4,67 & 5,91 & 0,46 & 0,61 & 1,39 & 2,37 \\
\hline $\mathrm{AM}$ & 2,32 & 3,71 & 5,58 & 6,70 & 0,79 & 1,07 & 1,69 & 2,69 \\
\hline RR & 2,94 & 3,98 & 5,64 & 7,17 & 1,05 & 1,53 & 2,83 & 3,27 \\
\hline PA & 2,44 & 3,51 & 4,80 & 5,93 & 1,02 & 1,43 & 2,01 & 2,84 \\
\hline $\mathrm{AP}$ & 3,02 & 4,38 & 6,55 & 7,48 & 1,21 & 1,47 & 2,23 & 3,51 \\
\hline MA & 1,08 & 1,93 & 3,62 & 5,02 & 0,45 & 0,72 & 1,28 & 2,27 \\
\hline PI & 1,1 & 2,08 & 3,80 & 5,01 & 0,33 & 0,59 & 1,26 & 2,22 \\
\hline $\mathrm{CE}$ & 1,45 & 2,55 & 4,27 & 5,73 & 0,35 & 0,59 & 1,19 & 2,33 \\
\hline RN & 1,64 & 2,93 & 55,04 & 6,59 & 0,51 & 0,78 & 1,52 & 2,68 \\
\hline $\mathrm{PB}$ & 1,36 & 2,50 & 4,29 & 5,58 & 0,43 & 0,67 & 1,19 & 2,20 \\
\hline $\mathrm{PE}$ & 2,03 & 3,20 & 5,12 & 6,48 & 0,44 & 0,68 & 1,46 & 2,62 \\
\hline $\mathrm{AL}$ & 1,23 & 2,22 & 4,05 & 5,29 & 0,32 & 0,51 & 1,15 & 2,18 \\
\hline SE & 1,41 & 2,75 & 4,69 & 5,85 & 0,38 & 0,66 & 1,46 & 2,46 \\
\hline $\mathrm{BA}$ & 1,56 & 2,57 & 4,27 & 5,79 & 0,45 & 0,65 & 1,33 & 2,45 \\
\hline MG & 2,77 & 4,24 & 5,93 & 7,29 & 1,20 & 1,88 & 3,01 & 3,97 \\
\hline ES & 2,79 & 4,46 & 6,27 & 7,45 & 1,28 & 2,16 & 3,47 & 4,43 \\
\hline RJ & 5,11 & 6,16 & 7,94 & 8,81 & 1,35 & 1,93 & 3,40 & 4,04 \\
\hline SP & 4,23 & 5,48 & 7,25 & 8,75 & 1,87 & 2,68 & 4,01 & 5,00 \\
\hline PR & 2,57 & 4,22 & 6,20 & 7,79 & 1,39 & 2,36 & 3,75 & 4,82 \\
\hline SC & 3,80 & 4,94 & 6,78 & 7,83 & 2,31 & 3,20 & 4,64 & 5,14 \\
\hline RS & 4,06 & 5,22 & 6,80 & 7,95 & 2,53 & 3,19 & 4,46 & 5,17 \\
\hline MT & 2,25 & 3,72 & 5,69 & 7,10 & 1,16 & 1,95 & 3,25 & 4,16 \\
\hline GO & 2,17 & 3,77 & 5,81 & 7,00 & 1,01 & 1,70 & 2,95 & 3,92 \\
\hline DF & 5,32 & 6,81 & 8,47 & 9,52 & 1,79 & 3,12 & 4,19 & 5,60 \\
\hline Média & 3,03 & 4,37 & 5,53 & 6,81 & 0,99 & 1,51 & 2,48 & 3,48 \\
\hline
\end{tabular}

Fonte: (a) Compilados pelos autores com base em Freitas e Bacha (2002, p. 14), que se basearam nos Censos Demográficos; (b) calculado com base no Censo Demográfico 2000.

O aumento da escolaridade média do trabalhador rural não foi suficiente para alterar fortemente o quadro de uso de mão-de-obra não qualificada na agropecuária. Istake \& Bacha (1999), considerando o Estado do Paraná, encontraram que os níveis de escolaridade têm aumentado entre os trabalhadores rurais, entretanto, a qualificação do trabalhador rural ainda é muito baixa. De acordo com os dados dos mencionados autores, em 1991, mais de $82 \%$ dos trabalhadores rurais do Paraná tinham cursado somente o ensino elementar. Esses resultados são coerentes com os obtidos para toda a agropecuária brasileira, mesmo considerando um estado líder na agropecuária, como o Paraná.

$\mathrm{O}$ aumento da educação média teve importantes reflexos sobre a evolução do PIB per capita brasileiro, conforme já enfatizado por Lau et al. (1993). Os resultados obtidos por Gonçalves et al. (1998) apontaram que o capital humano, em relação ao PIB per capita brasileiro, apresenta elasticidade maior do que um, 
demonstrando que as políticas de investimentos em educação no Brasil são eficazes.

O problema é que os retornos à educação e ao treinamento são diferentes quando se comparam os setores urbano e rural. Kassouf (1996) demonstrou que os retornos à educação foram maiores no setor urbano do que no setor rural, e que os retornos à educação foram superiores aos retornos ao treinamento. Dessa maneira, conclui essa autora, as pessoas residentes no setor urbano têm maiores incentivos a investir em educação e treinamento do que as pessoas residentes no setor rural. Para a população residente no meio rural, com grau de instrução mais elevado, o caminho esperado seria a migração para as cidades, especialmente para as médias e grandes cidades, na busca por melhores salários.

O nível médio de educação dos trabalhadores na agropecuária de todos os estados nordestinos é relativamente baixo em relação ao conjunto da nação. Em 1970, o nível de educação média nos estados nordestinos representava apenas 41\% da média nacional nesse mesmo setor. Os estados das Regiões Sudeste e Sul foram os que apresentaram as maiores médias de anos de estudo, em torno de $60 \%$ acima da média nacional. Apesar de ser crítica a situação da educação no meio rural, foi possível constatar que, em 1980, o patamar de educação média aumentou em todos os estados em torno de 50\%. Essa tendência de crescimento pode ser percebida também na década seguinte, com aumento em cerca de 64\%. Para período de 1991-2000, o crescimento foi de 40\%, em média. Os estados nordestinos aumentaram o nível médio de educação de seus trabalhadores agropecuários em 2000 para cerca de 68\% do nível médio de educação dos trabalhadores da agropecuária brasileira no seu conjunto. Em 1970, o nível médio de educação no setor rural dos estados da Região Centro-Oeste estava 33\% acima da média nacional nesse mesmo setor, subindo esse diferencial para 40\% em 1991, diferencial esse que se reduziu para $31 \%$ em 2000. Em termos relativos, observase que, em 1980 e 2000, os estados da Região Norte não conseguiram acompanhar, em termos do crescimento da escolaridade, o desempenho médio dos demais estados, no que se refere ao setor agropecuário. Em 1970, aquela região possuía três estados com a educação média superior à média nacional. Nos anos de 1980 e 1991, apenas dois estados (Roraima e Rondônia, nos quais se perceberam importantes melhoras nos níveis de educação dos seus trabalhadores rurais) se posicionaram quanto a essa variável acima da média nacional. Em 2000, esse número se manteve, trocando Roraima por Amapá. Entretanto, constatou-se maior equilíbrio quanto a essa variável entre os estados.

Os estados das Regiões Sul, Sudeste e Centro-Oeste apresentaram, nos quatro anos especificados na Tabela 4, níveis de educação média para os trabalhadores rurais superiores à média nacional no mesmo setor. Alguns estados como Rio Grande do Sul, Santa Catarina, São Paulo e Distrito Federal - chegaram a superar a média nacional em no mínimo 60\%, em 1991. Ao que tudo indica, e 
como têm mostrado as pesquisas relacionadas à teoria do capital humano, parte do desenvolvimento econômico desses estados é explicada pelo estoque de capital humano ali existente.

Comparando os valores das Tabelas 1 e 4, pode-se perceber que a riqueza e a pobreza dos estados estão, em grande medida, relacionadas às habilidades e conhecimento médio dos seus trabalhadores. Quanto mais conhecimento possuir um trabalhador, maior será o seu nível de abstração e mais dinâmico ele se torna no aprendizado e no desenvolvimento das tarefas. Com maior estoque de conhecimento, mais fácil seria também para os pequenos e médios agropecuaristas tomarem suas decisões de investimentos e de realocação de recursos perante as mudanças conjunturais. Para todo o Brasil, encontrou-se coeficiente de correlação entre os anos médios de educação em 2000 (última coluna da Tabela 4) e o VP por EH em 2000 (quarta coluna da Tabela 1) igual a 0,87. Esse coeficiente tem que ser analisado com cautela, pois o VP por EH é muito sensível aos preços dos produtos agropecuários. Isso explica o fato de essa alta correlação não ser verificada para 0 Nordeste e Norte para a mesma série de dados analisada.

\section{Conclusões}

Este trabalho mostrou que a agropecuária brasileira tem apresentado, de 1970 a 2000, desenvolvimento desigual em termos de estados, gerando grandes diferenças regionais.

Um indicador que chama a atenção relaciona-se ao crescimento do uso de capital na agropecuária brasileira no período de 1970 a 1995/96. A relação K/L vem crescendo praticamente em todos os estados brasileiros. Em alguns estados, esse crescimento foi mais intenso, como Acre, Roraima, Maranhão, Paraná, Santa Catarina e Mato Grosso. No entanto, o nível de tecnificação do setor agropecuário ainda é bastante desigual entre os estados brasileiros.

As estatísticas dos níveis de educação média na economia brasileira e na agropecuária, especificamente, revelam um quadro de forte desequilíbrio. A população rural, em 1970, era em grande parte analfabeta. No período de 1970 a 2000, verificou-se tendência de crescimento da escolaridade média no meio rural e de diminuição de sua diferença em relação à média brasileira. No entanto, o nível educacional na agropecuária ainda é baixo e muito desigual entre os estados brasileiros.

A diminuição das diferenças regionais do desenvolvimento da agropecuária suscita a discussão de quais mecanismos podem ser adotados para diminuí-las. Como mostrado ao longo do trabalho, o nível de desenvolvimento da agropecuária nos estados está em grande medida relacionado às habilidades e conhecimento médio dos seus trabalhadores e dos seus empresários. Dessa forma, 
o caminho inicial da diminuição das desigualdades passa por reduzir as diferenças regionais em termos de capital humano. Assim, as políticas de treinamento da mão-de-obra rural, tanto no ensino tradicional como no alternativo, devem ser formuladas de modo concomitante aos instrumentos que apenas estimulam a expansão da produção.

\section{Referências bibliográficas}

ARAÚJO, P. F. C.; SCHUH, G. E. Desenvolvimento econômico e o papel da agricultura. In: ECONOMIA e administração agroindustrial. Piracicaba: DESR/ESALQ, 1995. cap. 1, p. 1-28. (Série Didática, n. 96).

ARNADE, C. A. Productivity and technical change in Brazilian agriculture. New York: US Department of Agriculture. Economic Research Service, 1992. (Technical Bulletin number 1811).

ÁVILA, A. F. D.; EVENSON, R. E. Total factor productivity growth in the Brazilian agriculture and the role of agricultural research. In: CONGRESSO BRASILEIRO DE ECONOMIA E SOCIOLOGIA RURAL, 33, Curitiba, 1995. Anais... Brasília: Sober, 1995. p. 631-657.

BACHA, C. J. C.; ROCHA, M. T. O comportamento da agropecuária brasileira, no período de 1987 a 1996. Revista de Economia e Sociologia Rural, v. 36, n. 1, p. 35-59, jan./mar. 1998.

BARROS, A. L. M. Capital, produtividade e crescimento da agricultura: o Brasil de 1970 a 1995. Tese (Doutorado)-Escola Superior de Agricultura "Luiz de Queiroz", Universidade de São Paulo, Piracicaba, 1999. 149p.

BARROS, J. R. M.; GRAHAM, D. H. A agricultura brasileira e o problema da produção de alimentos. Pesquisa e Planejamento Econômico, v. 8, n. 3, p. 695-726, 1978.

BARROS, R. P.; MENDONÇA, R. S. P. de; DUARTE, R. P. N. Bem-estar, pobreza e desigualdade de renda: uma avaliação da evolução histórica e das disparidades regionais. Rio de Janeiro: Ipea, 1997. 60p. (Texto para Discussão, n. 454).

FREITAS, C. A. Contribuição do capital humano para o crescimento da agropecuária brasileira - período de 1970 a 1996. Tese (Doutorado)-Universidade de São Paulo, Piracicaba.

; BACHA, C. J. C. Análise do crescimento desigual do setor agropecuário brasileiro em termos de produtos e estados, período de 1970 a 1996. In: CONGRESSO BRASILEIRO DE ECONOMIA E SOCIOLOGIA RURAL, XL, Passo Fundo, 2002. Anais... Brasília: Sober, 1998. p. 211-221.

FERREIRA FILHO, J. B. S. Notas a respeito do desempenho agregado da agricultura brasileira no período 1980-1991. Revista de Economia e Sociologia Rural, v. 32, n. 3, p. 225-235, jul./set. 1994.

FURTADO, C. Desenvolvimento e subdesenvolvimento. Rio de Janeiro: Fundo de Cultura, 1961. 
GASQUES, J. G. Transformações estruturais da agricultura e a produtividade total dos fatores. In: GASQUES, J. G.; CONCEIÇÃO, J. C. (Org.). Transformações da agricultura e políticas públicas. Brasília: Ipea, 2001.

; CONCEIÇÃO, J. C. P. R. Crescimento da produtividade da agricultura brasileira. Brasília: Ipea, 1997. 21p. (Texto para Discussão, n. 520).

; VILLA VERDE, C. M. Crescimento da agricultura brasileira e política agrícola nos anos oitenta. Brasília: Ipea, 1990. 22p. (Texto para discussão, $\quad$ n. 204).

GONÇALVES, F. O.; SEABRA, F.; TEIXEIRA, J. R. O capital humano em um modelo de crescimento endógeno da economia brasileira. Análise Econômica, v. 16, n. 29, p. 139148, 1998.

ISTAKE, M.; BACHA, J. C. Comportamento do emprego na agropecuária do Paraná período de 1970 a 1996. Revista de Economia e Sociologia Rural, v. 37, n. 4, p. 71-101, 1999.

JONES, C. I. Introdução à teoria do crescimento econômico. São Paulo: Campus, 1999. $178 \mathrm{p}$.

KASSOUF, A. L. Retornos à educação e treinamento nos setores urbano e rural do Brasil. In: CONGRESSO BRASILEIRO DE ECONOMIA E SOCIOLOGIA RURAL, 34, Aracaju, 1996. Anais... Brasília: Sober, 1996. p. 771-783.

LAU, L. J. et al. Education and economic growth: some cross-sectional evidence from Brazil. Journal of Development Economics, v. 41, p. 45-70, 1993.

LAVINAS, L.; GARCIA, H. E.; AMARAL, M. R. de. Desigualdades regionais: indicadores socioeconômicos nos 90. Rio de Janeiro: Ipea, 1993. 83p. (Texto para discussão, n. 460).

MELLO, F. H. O crescimento agrícola brasileiro dos anos 80 e as perspectivas para os anos 90. Revista de Economia Política, v. 10, n. 3, p. 22-30, jul./set. 1990.

REZENDE, G. C. Política econômica e a agricultura na década de 80. In: CONGRESSO BRASILEIRO DE ECONOMIA E SOCIOLOGIA RURAL, 28, Piracicaba, 1989. Anais... Brasília: Sober, 1989. p. 284-309.

SILVA, G. L. S. P. Transforming Brazilian agriculture. In: CONGRESSO BRASILEIRO DE ECONOMIA E SOCIOLOGIA RURAL, 29, Campinas, 1991. Anais... Brasília: Sober, 1991. p. 254-278.

SILVA, J. G.; KAGEYAMA, A. Emprego e relações de trabalho na agricultura brasileira: uma análise dos dados censitários de 1960, 1970, e 1975. Pesquisa e Planejamento Econômico, v. 13, n. 1, p. 235-266, abr. 1983.

STADUTO, J. A. R.; FREITAS, C. A. Uma avaliação da mudança estrutural da produção agrícola brasileira no período 1959-1995. In: CONGRESSO BRASILEIRO DE ECONOMIA E SOCIOLOGIA RURAL, 39, Recife, 2001. Anais... Recife: Sober, 2001. (Cd-Room). 